\title{
The Relation Between Event Processing and the Duration of Emotional Experience
}

\author{
Philippe Verduyn, Iven Van Mechelen, and Francis Tuerlinckx \\ University of Leuven
}

\begin{abstract}
An emotional experience can last for only a couple of seconds up to several hours or even longer. In the present study, we examine to which extent covert intrapersonal actions (cognitions both related and unrelated to the emotion-eliciting stimulus) as well as overt interpersonal actions (social sharing) account for this variability in emotion duration. Participants were asked to report the duration of their anger, sadness, joy, and gratitude episodes on a daily basis during five days. Furthermore, information was collected with regard to their cognitions during the episodes and their social sharing behavior. Discretetime survival analyses revealed that for three of the four emotions under study, stimulus-related cognitions with the same valence as the emotion lead to a prolongation of the episode; in contrast, both stimulus-related and stimulus-unrelated cognitions with a valence opposite to the emotion lead to a shortening. Finally, for the four emotions under study, social sharing was associated with a prolongation. The findings are discussed in terms of a possible process basis underlying the time dynamics of negative as well as positive emotions.
\end{abstract}

Keywords: emotion duration, cognitive processing, valence, social sharing

Emotions change and evolve over time. Consequently, a full understanding of them can be reached only when their time-related aspects are captured adequately (e.g., Davidson, 1998; Eaton \& Funder, 2001; Hemenover, 2003; Schimmack, Oishi, Diener, \& Suh, 2000; Verduyn, Van Mechelen, Tuerlinckx, Meers, \& Van Coillie, 2009b). Surprisingly, however, the number of empirical studies focusing on dynamical properties of emotions is scarce.

A central time-related characteristic of emotions is the duration of emotional experience. Unlike a mood, an emotional experience is assumed to be elicited by a certain event and, as a result, to have a clear onset point (Beedie, Terry, \& Lane, 2005). The duration of an emotional episode can then further be defined as the amount of time between this onset point and the first moment the emotional experience is no longer felt (Verduyn, Delvaux, Van Coillie, Tuerlinckx, \& Van Mechelen, 2009a).

In previous studies, it has been shown that an emotional episode can last from only a couple of seconds up to several hours or even longer (Fitness \& Fletcher, 1993; Gilboa \& Revelle, 1994; Scherer, Walbott, \& Summerfield, 1986; Sonnemans, 1991; Verduyn et al., 2009a). As explained below, part of this variability in duration has been accounted for by a number of trait, episode, and moment predictors.

Trait predictors are predictors that are stable both within and across emotional episodes. On an empirical level, a number of traits have been examined as possible determinants of duration

Philippe Verduyn, Iven Van Mechelen, and Francis Tuerlinckx, Department of Psychology, University of Leuven, Belgium.

The present research was supported by Grant GOA/05/04 and Grant GOA/10/02 from the Research Fund of the University of Leuven.

Correspondence concerning this article should be addressed to Philippe Verduyn, University of Leuven, Department of Psychology, Tiensestraat 102, 3000 Leuven, Belgium. E-mail: philippe.verduyn@psy.kuleuven.be including the Big Five and suppression tendency (Schimmack, 2003; Verduyn et al., 2009a). However, most relations between these traits and duration did not replicate across studies.

Episode predictors are predictors that are constant within a certain emotional episode but that may vary across episodes. In previous research, a number of episode predictors of the duration of emotional experience have been identified. In particular, it has been shown that the higher the importance of the eliciting stimulus and the higher the intensity of the emotion at onset, the longer the episode lasts (Schimmack, 2003; Sonnemans \& Frijda, 1995; Verduyn et al., 2009a).

Finally, moment predictors are predictors that may fluctuate within an emotional episode. Regarding this class of predictors, it was found that reappearances of the eliciting stimulus during an episode may lead to a prolongation of the emotional experience (Sbarra, 2006; Verduyn et al., 2009a). It is important that such reappearances do not necessarily have to be physical, as mental reappearances (i.e., thinking back at the eliciting stimulus) may lead to a prolongation as well (Verduyn et al., 2009a).

In sum, previous studies have revealed a number of determinants of the duration of emotional experience. By means of the present study we first want to replicate some of these findings. Particularly, we want to replicate the positive relation between the importance of the eliciting event and initial emotional intensity on the one hand and duration on the other hand. More important, beyond replicating previous findings, we aim to extend our understanding of emotion dynamics by focusing on a potentially important class of determinants of emotion duration. In particular, we will examine the impact of actions one may undertake after the emotion has been elicited in line with previous work on mental reappearances of the eliciting stimulus (Verduyn et al., 2009a). The relevance of such actions appears, for example, from the fact that people generally do not passively undergo their emotions; rather they often actively influence their emotional experiences as 
studied within the emotion regulation literature (e.g., Gross, 2007). Obviously, such regulatory actions may also have an impact on the duration of emotional episodes. In the present paper, we will focus on both covert as well as overt actions. Concerning covert actions, we will examine thoughts both related and unrelated to the eliciting stimulus, whereas with regard to overt actions, we will examine social sharing. Below, we will discuss these actions more in detail along with specific hypotheses regarding their relation with duration.

\section{Thoughts}

\section{Thoughts Related to the Eliciting Stimulus}

As appears from previous results on mental reappearances of emotion eliciting stimuli mentioned above, thoughts related to the eliciting stimulus during an emotional episode may influence emotion duration. Particularly, it has been found that such reappearances may lead to a prolongation of the emotional experience (Verduyn et al., 2009a).

A limitation of the study by Verduyn et al. (2009a), however, is that only the mere mental presence versus absence of the eliciting stimulus was taken into account and that no attention was given to the specific content of the thoughts. The content of thoughts related to the eliciting stimulus may differ in many aspects and it is rather obvious that this may affect the relation with duration. A key feature in this regard may be the valence of the thought content. For example, during an anger episode caused by an insult one may have both negative thoughts such as "he really enjoys hurting me," and positive thoughts such as "he probably did not mean it that way." Obviously, the two types of thoughts can be expected to have quite a different impact on the emotional episode. In this regard, in previous studies on rumination and reappraisal, it has been shown that ruminating on the negative aspects of an unfavorable emotional event leads to an increase in negative affect (Bushman, 2002; McLaughlin, Borkovec, \& Sibrava, 2007; Rude, Maestas, \& Neff, 2007), while reappraising the event in a positive way leads to a decrease (Gross, 1998; Ray, Wilhelm, \& Gross, 2008; Dandoy \& Goldstein, 1990). This means that depending on thought valence, emotional intensity may increase or decrease.

It remains, however, an open question whether these findings extend to the duration of emotional episodes. Intensity and duration are related to a certain degree (Sonnemans \& Frijda, 1994; Verduyn et al., 2009a), but one may not simply assume that factors determining the former will also determine the latter (Frijda, 2007).

A second open question relates to the fact that up to now the effects of cognitive processing on emotional episodes have been studied almost exclusively with regard to negative emotions. There is, however, no a priori reason why similar effects would not show up within the context of positive emotions, too.

In summary, a first major goal of the present study is to investigate whether the effect of thoughts related to the emotioneliciting stimulus on the duration of emotional experience will depend on thought valence, and this in case of both positive and negative emotions. In particular, we hypothesize that: (1) for negative emotions negative thoughts about the eliciting stimulus will lead to a prolongation of the emotional episode, while positive thoughts will lead to a shortening, whereas (2) for positive emo- tions positive thoughts lead to a prolongation and negative thoughts to a shortening.

\section{Thoughts Unrelated to the Eliciting Stimulus}

Thoughts during the emotional episode unrelated to the eliciting stimulus may also influence the emotional episode. In particular, previous studies on distraction indicate that thinking about something unrelated to the elicitor may be an effective strategy (at least in the short term) to handle negative emotional events, as it may lead to a decrease in intensity of negative affect (Fennell \& Teasdale, 1984; Morrow \& Nolen-Hoeksema, 1990). Distraction will only be successful, however, when appropriate distracters are used. In particular, distraction has appeared to be an effective strategy to cope with negative emotions when positive distracters are employed, whereas negative distracters do not lead to any intensity reduction at all (Wenzlaff, Wegner \& Roper, 1988; Boden \& Baumeister, 1997).

$\mathrm{tk}$;2In line with these findings on the effect of distraction on emotion intensity, one may wonder whether thinking about something unrelated to the elicitor will also influence emotion duration. Moreover, previous studies on the effect of distraction focused exclusively on negative (mainly depressive) moods and emotions. Distraction, however, could also affect positive emotions, in particular in case the distracters are negative in valence (e.g., when confronted with something funny at a funeral one may shift attention to negative distracting thoughts to suppress the positive emotion).

Summarizing, a second central aim of the current study is to examine whether thoughts that are unrelated to the eliciting stimulus influence the duration of both positive and negative emotional episodes. In particular, we hypothesize that (1) in case of negative emotions, thinking about a positive distracter will lead to a shortening of the episode while negative distracting thoughts will show no effect, and (2) in case of positive emotions, negative distracters will lead to a shortening while positive distracters will not affect duration.

\section{Social Sharing}

Social sharing is one of the most often applied emotion-driven overt actions. Indeed, it has been shown that people undertake sharing at some point in time following the emotional event in $80-95 \%$ of the cases (Rimé, Mesquita, Philippot \& Boca, 1991). Depending on the nature of the emotion, social sharing may enhance the emotional experience or not. Particularly, it has been shown that social sharing of positive events leads to an increase in the intensity of the associated positive emotions (Langston, 1994; Gable, Reis, Impett, \& Asher, 2004) while sharing following negatives events has no effect on the intensity of the associated negative emotions (Finkenauer \& Rimé, 1998; Zech \& Rimé, 2005).

As the previous findings pertain to the relation between social sharing and emotional intensity, one may wonder whether similar relations exist between social sharing and emotion duration. Therefore, a third central aim of the present study is to investigate whether sharing positive events will lead to a prolongation of the corresponding positive emotional episode while sharing negative events will not affect the duration of the corresponding negative episode. 


\section{Overview of the Present Study}

By means of the present study, we want to replicate the positive relation between the importance of the eliciting stimulus and the initial emotional intensity on the one hand and duration on the other hand. More important, we aim to extend previous findings by testing the validity of the following hypotheses: (1) Thoughts related to the eliciting stimulus during the emotional episode lead only to a prolongation of the episode if the valence of the thought is the same as the valence of the emotion. In case of opposite valences, thoughts will lead to a shortening. (2) Thoughts that are unrelated to the eliciting stimulus during the emotional episode lead only to a shortening of the episode if the valence of the thought is opposite to the valence of the emotion; otherwise, no effect on duration is expected. (3) Social sharing of positive events leads to a prolongation of the associated positive emotion while sharing negative events has no effect on the duration of the associated negative emotion.

To test the hypotheses described above, we performed a large scale experience sampling study (Kahneman, Krueger, Schkade, Schwarz, \& Stone, 2004; Bolger, Davis, \& Rafaeli, 2003) on the duration of episodes of joy, gratitude, anger, and sadness. This set of emotions was chosen as it contains two positive as well as two negative emotions. Moreover, it was also used in another recent study on emotion duration (i.e., Verduyn et al., 2009a) and therefore allows for a straightforward comparison of results.

\section{Method}

\section{Participants}

Participants were 344 students of the University of Leuven, 71 men and 273 women. Their mean age was $19(S D=3.3)$. They participated in partial fulfillment of a course requirement. Twentyfour participants did not complete the experience sampling questionnaire each day of the study. However, as the results were not affected by the data of these participants, they were not excluded from the final sample.

\section{Materials and Procedure}

Participants were invited to come to the psychology department in small groups of 20 to 30 people. They were informed that they were taking part in a study on emotional experience that would last for five consecutive days starting on the evening of the day they received the instructions. Next, they were explained that each evening right before going to bed they would have to fill out a questionnaire on the emotions they had experienced during that day. To make sure that they would supply information regarding emotional episodes and not moods, participants were asked to report only episodes that were clearly elicited by a certain internal or external event. Furthermore, participants were explained that an emotional episode was defined to end as soon as the emotion is not longer felt for the first time; in case the emotion is experienced again later on, they were asked to consider this as a new episode. Subsequently, they were provided the web address where the daily questionnaire could be found.

The daily questionnaire was divided into four blocks, each block corresponding to one of the four emotions under study. The order of these four blocks was randomized (i.e., each day participants received a new random sequence among the four blocks). In order not to overload the participants, they were only presented questions until they had provided information with regard to two episodes. In case participants had experienced at most only a single episode of one of the four emotions, filler questions were asked about other emotions until information regarding two episodes was given. The specific questions on the emotional episodes will be described below. This will be done for anger episodes; the questions on the other emotions (including the filler emotions) were identical.

Participants first answered how often they had experienced anger that day $(0,1,2$, more than two times). Next, they were asked a set of questions that were repeated for the second anger episode, if this had taken place. In case participants experienced more than two anger episodes, questions were asked with regard to the two most recent episodes only.

For each anger episode, participants were asked to rate on an 8-point Likert scale: (1) the importance of the event or situation that elicited the emotional episode $(0=$ not important at all to $7=$ very important), and (2) the intensity of the emotion at the beginning of the emotional episode $(0=$ not intense at all to $7=$ very intense). Next, they had to indicate whether they had talked about the eliciting event (after the onset of the emotion) with someone who was not (directly) involved in the emotional event (yes/no). Subsequently, they had to rate the duration of the emotional episode. For this purpose, a bar was presented, which was divided into six intervals. The total length of the bar denoted 60 minutes, each interval representing 10 minutes. Participants had to indicate in which of the six intervals their emotional episode had ended. If the episode lasted for longer than one hour, they had to put a cross in a checkbox, labeled "the emotion took longer than one hour." Finally, they had to indicate with yes/no for each interval in which their emotional episode was still ongoing whether they had (1) positive thoughts about the eliciting stimulus, (2) negative thoughts about the eliciting stimulus, (3) positive thoughts about something unrelated to the eliciting stimulus, and (4) negative thoughts about something unrelated to the eliciting stimulus. This implies that participants were given the opportunity to indicate multiple kinds of thoughts during a specific time-interval.

\section{Data Analysis}

The data set contains information regarding the duration of emotional episodes as well as possible episode and moment predictors of duration. To analyze these data, standard statistical techniques such as regression analysis cannot be readily applied because the exact duration of some emotional episodes (viz., the episodes that last longer than the registration window of 1 hour) is not known, and because the value of moment predictors may change within emotional episodes. Therefore, the data were analyzed using discrete-time survival analysis, which is well-suited to describe and model durational data (Singer \& Willett, 2003). This type of analysis relies on three central statistics, namely the hazard rate, the survivor rate, and median lifetime.

The hazard rate is the conditional probability that an emotional episode that has not yet ended at the beginning of a certain interval, will end during that interval; it can be calculated as the number of episodes that ended in an interval under study divided by the 
number of episodes that were still ongoing at the beginning of that interval. The survivor rate is the probability that an emotional episode will still be alive at the end of a specific interval; this survivor rate can be computed as the number of emotional episodes that are still ongoing at the end of a given interval divided by the total number of emotional episodes. Finally, the median lifetime pertains to the point in time at which half of the emotional episodes under study have ended. Each of these statistics (i.e., hazard rate, survivor rate, and median lifetime) was calculated for each emotion separately on the basis of all episodes of the emotion in question from all individuals.

In discrete-time survival analysis, the logit of the hazard rates is modeled in terms of a weighted sum of predictors, including both substantive predictors and number of dummy variables equal to the number of time intervals. Together, the dummy variables represent the baseline hazard function. Depending on the sign of the weight of a substantive predictor, a higher score on it leads to an upward or downward shift of the baseline hazard function. In the results section, to ease the interpretation, all predictor weights will be reflected such that a positive weight means that a higher score on the predictor is associated with longer duration, whereas the reverse holds for negative weights.

In the present analysis, all substantive predictors were first analyzed separately. In a second analysis, all predictors were included in the model simultaneously to examine the contribution of each predictor while controlling for the effect of the others. In both types of analyses we included a random intercept to capture dependencies due to each participant reporting multiple emotional episodes; through such a random intercept the overall level of the baseline hazard is allowed to vary across persons.

\section{Results}

\section{Hazard and Survivor Functions}

The hazard functions of joy, gratitude, anger, and sadness are presented in Figure 1. On the whole, the hazard rates are fairly high during the first time-intervals and subsequently decline over time. This implies that, on the one hand, a relatively large number of emotional episodes end during the first intervals and that, on the other hand, episodes that survived the first intervals are often long lasting ones, as the conditional probability that an emotional episode ends during the last intervals is comparatively low.

Next to this general pattern, there are some differences between the hazard functions of the four emotions. A first difference is that the hazard rate of gratitude is higher at the first two intervals than that of the other emotions under study. This indicates that short episodes (i.e., shorter than 20 minutes) of gratitude are more common than short episodes of joy, anger and sadness. Second, over the entire period the hazard rate of sadness is lower than that of the other emotions; this indicates that sadness is a relatively long lasting emotion.

The survivor functions are presented in Figure 2. In general, they show a steep drop during the first 30 minutes and a smaller decline thereafter, which links up with the relatively high values of the hazard functions in the first three intervals. On average, $80 \%$ of the emotional episodes ends within the first 30 minutes, whereas during the next 30 minutes only an additional $10 \%$ ends.

As regards differences between the survivor functions of the different emotions, the survivor function of gratitude drops steeper than those of anger and joy, which in turn drop more steeply than the survivor function of sadness. These differences are further also reflected in the differences in the median lifetimes of the emotions under study, which are 9 minutes for gratitude, 12 minutes for anger as well as for joy, and 15 minutes for sadness. All this links up with the differences in the hazard functions described above.

Besides differences in hazard and survivor functions between emotions, there is also evidence for differences in the overall level of these functions between persons: For each of the four emotions under study, the variance in the baseline hazard functions across persons is significantly different from zero (Joy: $\chi_{(1)}^{2}=35.64, p<.001$; Gratitude: $\chi_{(1)}^{2}=19.06, p<.001$; Anger: $\chi_{(1)}^{2}=11.32, p<.001$; Sadness: $\left.\chi_{(1)}^{2}=14.52, p<.001\right)$. This implies that for each of the four emotions some people tend to experience longer episodes than others.

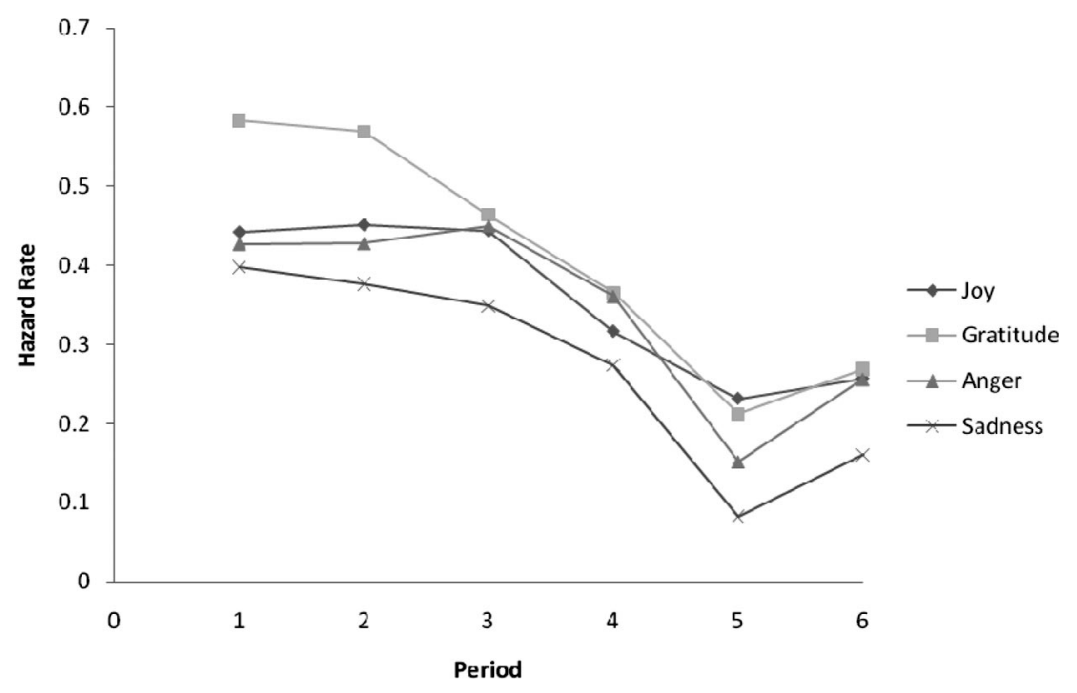

Figure 1. Hazard functions of joy, gratitude, anger, and sadness. 


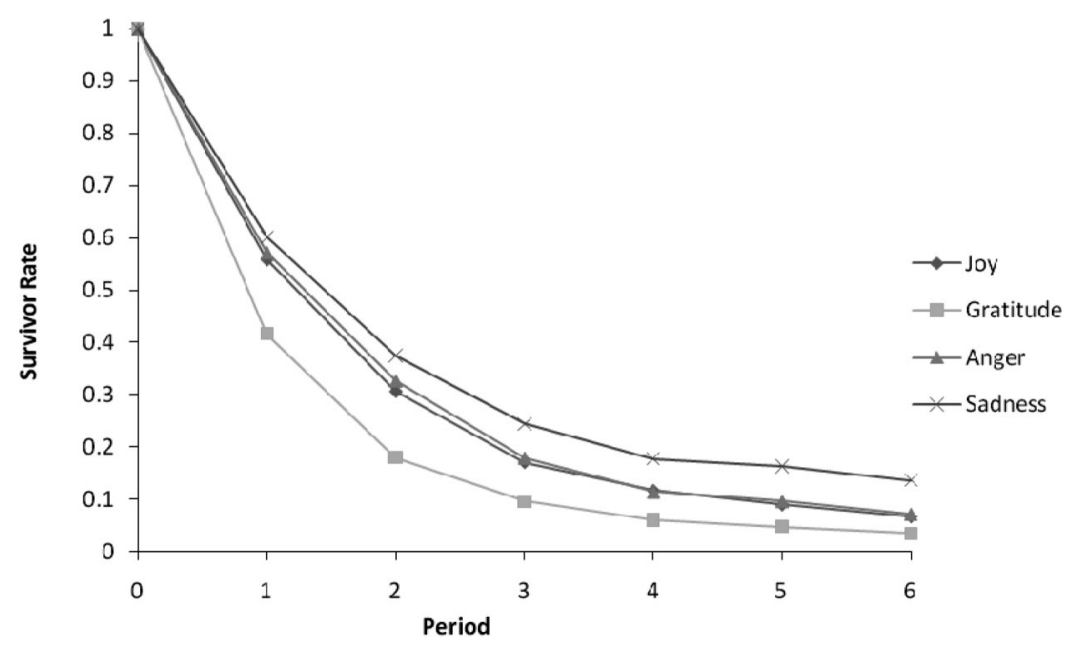

Figure 2. Survivor functions of joy, gratitude, anger, and sadness.

\section{Predictors of Duration: Descriptive Statistics}

The mean and standard deviation of the predictors of duration as included in our study are presented in Table 1. Episodes were on average elicited by a stimulus of moderate importance and had a moderate level of intensity at onset. Moreover, from the standard deviations (in conjunction with the moderate mean level) it can be inferred that the data include events of high as well as of low importance, and episodes of high as well as of low initial intensity. With regard to thoughts during the emotional episodes, it appears that thoughts related to the eliciting event showed up more often than thoughts about issues that are unrelated to the elicitor. Moreover, people tend to have more thoughts of the same valence as the emotion than thoughts of opposite valence. Regarding social sharing, in almost $40 \%$ of the episodes participants talk about the emotional event during the day in which the episode in question occurred.

Besides this general pattern, a number of differences between the emotions can be observed. First, the importance of the eliciting stimulus and the initial intensity of the emotion is highest for sadness. Second, thoughts related to the eliciting stimulus with a valence opposite to the valence of the emotion are more frequent during negative than during positive emotions. Third, thoughts unrelated to the elicitor with the same valence as the valence of the emotion are more frequent during positive emotions than during negative ones, while the reverse holds for unrelated thoughts with an opposite valence. Finally, participants talked more often with a third party about events that elicited negative emotions than about events that elicited positive emotions.

\section{Relation Between Predictors and Duration}

To study whether and to which extent the predictors of duration included in our study account for the observed variability in episode duration, a series of simple and multiple discrete-time survival analyses are performed. The weights of the substantive predictors of joy, gratitude, anger, and sadness obtained from these analyses are presented in Table 2. Recall that a positive (respectively negative) weight means that a higher score on the predictor is associated with a longer (respectively shorter) duration. For example, a positive weight for "the importance of the eliciting stimulus" implies that the more the eliciting stimulus is perceived as important, the longer the emotional episode lasts. With a few exceptions, the analyses involving each predictor separately and all

Table 1

Mean and Standard Deviation of the Predictors of the Duration of Joy, Gratitude, Anger, and Sadness

\begin{tabular}{|c|c|c|c|c|c|c|c|c|}
\hline \multirow[b]{2}{*}{ Predictor } & \multicolumn{2}{|c|}{ Joy } & \multicolumn{2}{|c|}{ Gratitude } & \multicolumn{2}{|c|}{ Anger } & \multicolumn{2}{|c|}{ Sadness } \\
\hline & Mean & $S D$ & Mean & $S D$ & Mean & $S D$ & Mean & $S D$ \\
\hline Importance of the eliciting situation ${ }^{\mathrm{a}}$ & 4.23 & 1.79 & 4.27 & 1.78 & 4.10 & 1.99 & 4.92 & 1.77 \\
\hline Intensity of the emotion at onset ${ }^{a}$ & 4.33 & 1.71 & 3.97 & 1.73 & 4.51 & 1.86 & 4.63 & 1.76 \\
\hline \multicolumn{9}{|l|}{ Thoughts related to the eliciting event } \\
\hline Same valence ${ }^{\mathrm{b}}$ & 0.89 & 0.32 & 0.86 & 0.35 & 0.84 & 0.37 & 0.80 & 0.40 \\
\hline Opposite valence ${ }^{\mathrm{b}}$ & 0.09 & 0.28 & 0.10 & 0.30 & 0.16 & 0.37 & 0.27 & 0.44 \\
\hline \multicolumn{9}{|l|}{ Thoughts unrelated to the eliciting event } \\
\hline Same valence ${ }^{\mathrm{b}}$ & 0.49 & 0.50 & 0.53 & 0.50 & 0.25 & 0.43 & 0.28 & 0.45 \\
\hline Opposite valence $^{\mathrm{b}}$ & 0.14 & 0.34 & 0.17 & 0.37 & 0.35 & 0.48 & 0.33 & 0.47 \\
\hline Social sharing ${ }^{\mathrm{a}}$ & 0.35 & 0.48 & 0.32 & 0.47 & 0.50 & 0.50 & 0.42 & 0.49 \\
\hline
\end{tabular}

${ }^{a}$ As these are episode predictors, the mean is calculated across episodes. ${ }^{\mathrm{b}}$ As these are moment predictors the mean is calculated across time-intervals. 


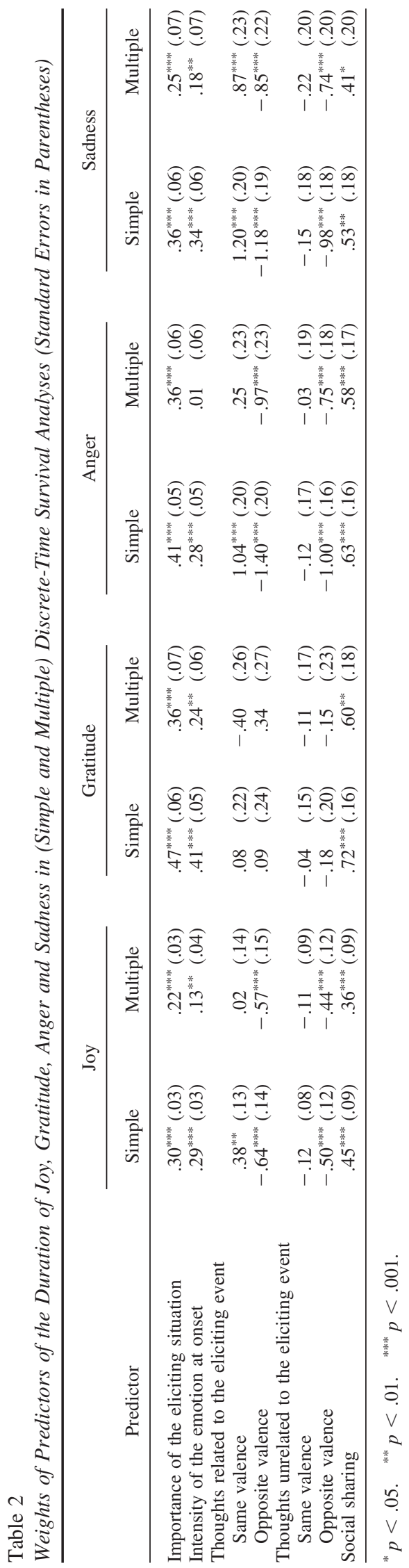

predictors simultaneously lead to the same conclusions. This means that, if a predictor contributes to explaining variability in episode duration, its contribution is almost always also a distinctive one. Below, we will discuss the contributions of the different groups of predictors as included in the study somewhat more in detail.

Regarding the predictors pertaining to the moment of emotion elicitation (i.e., the importance of the eliciting stimulus and the intensity of the emotion at onset), a consistent pattern across emotions is found. In particular, the higher the importance of the eliciting stimulus and the higher the initial intensity, the longer the emotional episode lasts.

Concerning thoughts during the emotional episode, a number of significant relations with duration showed up for all emotions under study except for gratitude. In particular, for joy, anger, and sadness, thoughts with a valence opposite to the valence of the emotion lead to a shortening of the emotional episode, and this holds for thoughts that are either related or unrelated to the eliciting stimulus. In contrast, thoughts with the same valence as the emotion may lead to a prolongation of the emotional episode; however, this is only the case if the thought is related to the eliciting stimulus. All this implies that thinking back at the eliciting event may not only prolong but also shorten the emotional episode, the key in determining the direction of the effect being the correspondence between the valence of the cognitions and the valence of the emotion. Furthermore, thinking about something unrelated to the eliciting event (which may be considered a kind of distraction) may sometimes lead to an episodes shortening; this, however, is only the case if the valence of the thoughts is opposite to the valence of the emotion.

Finally, social sharing appears to be positively related to emotion duration. Moreover, this is the case for all emotions under study, both positive and negative ones. Apparently, talking about the eliciting stimulus keeps the emotional event and corresponding emotion active. This, in turn, may then cause the emotional episode to last longer.

\section{Discussion}

This study provided evidence that the duration of emotional experience is highly variable. On the one hand, there are overall differences between emotions in that episodes of gratitude are in general shorter than episodes of anger and joy, which in turn are shorter than episodes of sadness. This finding largely replicates the order of emotions with regard to duration as found in previous studies (Scherer et al., 1986; Verduyn et al., 2009a); the only exception is that of anger episodes which, in contrast to earlier findings, do not tend to be shorter than episodes of joy. (The latter might be due to the large number of women in the present sample). On the other hand, duration is also highly variable within emotions, as for each of the four emotions under study episodes may be relatively short (i.e., shorter than 10 minutes) as well as relatively long (i.e., longer than one hour).

More important, besides showing that duration is highly variable, we were able to identify a number of factors that may account for this variability. First, we replicated the effects of two determinants of duration found in previous research (Schimmack, 2003; Sonnemans \& Frijda, 1995; Verduyn et al., 2009a): The importance of the eliciting stimulus and initial intensity of the emotion, 
with for each of the four emotions under study, the higher the importance of the eliciting stimulus and the higher the initial intensity, the longer the emotional episode lasts. Regarding the latter, it should be stressed that this finding does not imply a relation between duration and overall emotion intensity, which, in previous studies has been found to be hardly related to duration (Sonnemans \& Frijda, 1994; Frederickson \& Kahneman, 1993).

Yet, the fact that the eliciting event plays a major role does not imply that people merely passively undergo the event and the corresponding emotion. In particular, the present study extended previous research on determinants of emotion duration by showing that the way one deals with the eliciting event, at a covert cognitive as well as an overt interpersonal level, may affect the duration of emotional experience. Below, we will discuss these findings more in detail.

\section{Thoughts}

Thoughts related to the eliciting stimulus. Verduyn et al. (2009a) have shown that mental reappearances of the eliciting stimulus during an emotional episode lead to a prolongation of the episode. In the present study, we showed that thinking back at the eliciting event may not only lead to a prolongation but to a shortening as well. The key factor that accounts for the direction of the effect is the correspondence between the valence of the thought and the valence of the emotion. In case stimulus-related cognitions have the same valence as the emotion, thoughts appear to act as fuel that stirs up the emotional fire and leads to a prolongation of the episode. This may be due to the fact that such cognitions line up with the initial appraisals that caused the emotion, which, as a result, may be strengthened; in turn this may lead to a prolongation of the emotional episode. ${ }^{1}$ In contrast, if during an emotional episode one has stimulus-related thoughts of an opposite valence, such thoughts may extinguish the emotional fire and lead to a shortening of the episode. This might be due to a reappraisal of the emotion-eliciting event. These relations hold for each emotion under study except for gratitude. One may speculate that the latter finding could be accounted for by the somewhat more volatile nature of gratitude (as appears, e.g., from its relatively short duration), which may imply a smaller impact of cognitions on the emotion in question.

Before continuing this discussion, two important remarks are to be made. First, although stimulus-related cognitions can both prolong and curtail emotional episodes, both types of effects do not show up equally often. This is because people more often experience stimulus-related thoughts with the same valence as the emotion under study. Otherwise, this may also explain why in previous research (in which thought valence was not taken into account) thinking back at the elicitor was associated with episode prolongation (Verduyn et al., 2009a).

Second, in the past the effect of stimulus-related thoughts has been studied almost exclusively within the context of negative emotions. For such emotions it has been found that thoughts with the same valence (or rumination) lead to an intensification (Bushman, 2002; McLaughlin, Borkovec, \& Sibrava, 2007; Rude, Maestas, \& Neff, 2007) while thoughts with an opposite valence (or reappraisal) lead to a reduction of emotional intensity (Gross, 1998; Ray, Wilhelm, \& Gross, 2008; Dandoy \& Goldstein, 1990). From a hedonistic point of view, one has therefore argued that reappraisal is an adaptive strategy whereas rumination is not (e.g., Nezlek \& Kuppens, 2008). Yet, in the present study, we found that for a positive emotion (joy) too, stimulus-related thoughts with the same valence (i.e., "rumination") lead to a prolongation whereas thoughts with an opposite valence (or reappraisal) lead to a shortening. From a hedonistic point of view, in the case of joy one could consider "rumination," unlike reappraisal, adaptive. All this means that labeling some type of thinking as adaptive or maladaptive is of no relevance to understand the mechanisms underlying the shortening or prolongation of emotional episodes.

Thoughts unrelated to the eliciting stimulus. During an episode, one may also think about issues that are unrelated to the emotion-eliciting event (i.e., distraction). In the past, the effect of distraction on emotion duration has hardly been examined. In the present study, we have shown that in some cases distracting thoughts may affect emotion duration. Whether this is the case, indeed, depends on the correspondence between the valence of the thought and the valence of the emotion. In particular, whereas unrelated thoughts with the same valence do not affect episode duration, unrelated thoughts with opposite valence appear to lead to a shortening. One should note that the latter effect cannot be readily explained by an effect of distracting thoughts on the content of the appraisals linked to the emotion under study (as was the case for related thoughts), as distracting thoughts have nothing to do with the stimulus that elicited the emotion. One may conjecture, however, that unrelated thoughts with an opposite valence may lead to an emotion with a valence opposite to that of the emotion under study. This could imply a conflict as people may have difficulties experiencing simultaneously positive and negative emotions (which are typically associated with incompatible action tendencies; Brehm, 1999; Russell \& Carroll, 1999; Russell $\&$ Feldman-Barrett, 1999). To resolve this conflict, the intensity of the original emotion may go to zero which, given our definition, implies the end of the emotional episode in question. In contrast, in case unrelated thoughts do not have an opposite valence, they will not be disturbing as they do not interfere with the ongoing emotion.

It may be noted that previous studies on the effect of unrelated thoughts (or distraction) have mainly focused on negative emotions. For such emotions it has been found that unrelated thoughts with an opposite valence lead to a decrease in intensity; this implies that, from a hedonistic point of view, distraction may be considered as an adaptive strategy (Wenzlaff, Wegner, \& Roper, 1988; Boden \& Baumeister, 1997). However, in the present study a distracting effect has also been found for the duration of joy, as distracters with negative valence have been shown to lead to a shortening of joy episodes; from a hedonistic point of view, such a shortening could be considered maladaptive. Again, our findings reveal a common pattern for negative and positive emotions; whether or not an action such as looking for distraction is adaptive is only of secondary importance.

\footnotetext{
${ }^{1}$ The hypothesis that thinking (and also sharing) lead to a prolongation because these processes take time is contradicted by the finding that thoughts with a valence opposite to the emotion lead to a shortening.
} 


\section{Social Sharing}

People may not only process an emotional event at an intrapersonal level but also by sharing it with others. Common sense suggests that, especially in the case of negative emotions, social sharing is a beneficial coping strategy as it may help to "get it off your chest." Many psychologists share this view and believe that merely talking about the emotional event may dissolve its emotional impact (Scheff, 1979). However, empirical findings do not line up with this "expression-discharge" hypothesis. In particular, in previous studies it has been found that sharing does not affect the intensity of negative emotions (Finkenauer \& Rimé, 1998; Zech \& Rimé, 2005) and may even lead to an increase of intensity in some cases (Langston, 1994; Gable et al., 2004).

The present study provides further evidence that social sharing does not lead to a down regulation of emotional experiences, in that social sharing appears to be associated with a prolongation of both positive and negative emotions. To better understand the effect of social sharing on emotion duration, our findings on stimulus-related cognitions might be helpful. Social sharing, indeed, pertains, almost by definition, to the eliciting stimulus. One may further conjecture (Rimé, 2009) that the partner(s) in a social sharing endeavor will often not stimulate a cognitive reframing of the eliciting event but rather will provide an opportunity to vent the emotional experience. As a consequence, the valence of the conversation can be assumed to correspond quite often to that of the emotion experienced. Similar to thoughts related to the eliciting stimulus with the same valence, this may finally lead to a prolongation of the emotional episode under study. To get a better understanding of the relation between those different types of sharing and duration, however, sharing would have to be measured as a moment predictor. Otherwise, this would also guarantee that the actual sharing takes place during the emotional episode, which in the present study was not controlled for.

\section{Conclusion}

The duration of emotional experience is highly variable. A factor that accounts for a significant part of this variability is the eliciting event, as both the perceived importance and the initial intensity of the emotion it elicits are major determinants of episode duration. However, one does not have to merely passively undergo an emotion eliciting event and the corresponding emotion. Intrapersonal as well as interpersonal processes after the emotion onset may influence the duration of the emotional experience. In particular, one may actively prolong an emotion by means of thoughts with a valence equal to that of the emotion, or shorten emotion duration by shifting perspective and seeing the good in the bad or vice versa. Moreover, one may also shorten the duration of an emotional episode by diverting attention away to topics unrelated to the emotional event, provided that the valence of the distracter is opposite to the valence of the emotion. Finally, one may also prolong an emotional experience by talking about what happened to others. It is important to note that the effect of the actions mentioned above is not restricted to negative emotions as social sharing also prolongs episodes of joy and gratitude whereas shortening and prolongation effects of thoughts were also found for joy. Also, whether thinking or social sharing, from a hedonistic point of view, lead to adaptive or maladaptive outcomes, appears to be only of secondary importance to understand the processes underlying the duration of emotional episodes.

\section{References}

Beedie, C. J., Terry, P. C., \& Lane, A. M. (2005). Distinctions between emotion and mood. Cognition and Emotion, 19, 847-878.

Boden, J. M., \& Baumeister, R. F. (1997). Repressive coping: Distraction using pleasant thoughts and memories. Journal of Personality and Social Psychology, 73, 45-62.

Bolger, N., Davis, A., \& Rafaeli, E. (2003). Diary methods: Capturing life as it is lived. Annual Review of Psychology, 54, 579-616.

Brehm, J. W. (1999). The intensity of emotion. Personality and Social Psychology Review, 3, 2-22.

Bushman, B. J. (2002). Does venting anger feed or extinguish the flame? Catharsis, rumination, distraction, anger, and aggressive responding. Personality and Social Psychology Bulletin, 28, 724-731.

Dandoy, A. C., \& Goldstein, A. G. (1990). The use of cognitive appraisal to reduce stress reactions: A replication. Journal of Social Behavior and Personality, 5, 275-285.

Davidson, R. J. (1998). Affective style and affective disorders: Perspectives from affective neuroscience. Cognition and Emotion, 12, 307-330.

Eaton, L. G., \& Funder, D. C. (2001). Emotional experience in daily life: Valence, variability, and rate of change. Emotion, 1, 413-421.

Fennell, M. J., \& Teasdale, J. D. (1984). The effects of distraction on thinking and affect in depressed patients. British Journal of Clinical Psychology, 23, 65-66.

Finkenauer, F., \& Rimé, B. (1998). Socially shared emotional experiences vs. emotional experiences kept secret: Differential characteristics and consequences. Journal of Social and Clinical Psychology, 17, 295-318.

Fitness, J., \& Fletcher, G. J. O. (1993). Love, hate, anger, and jealousy in close relationships: A prototype and cognitive appraisal analysis. Journal of Personality and Social Psychology, 65, 942-958.

Fredrickson, B. L., \& Kahneman, D. (1993). Duration neglect in retrospective evaluations of affective episodes. Journal of Personality and Social Psychology, 65, 45-55.

Frijda, N. H. (2007). The laws of emotion. Mahwah: Erlbaum.

Gable, S. L., Reis, H. T., Impett, E. A., \& Asher, E. R. (2004). What do you do when things go right? The intrapersonal and interpersonal benefits of sharing positive events. Journal of Personality and Social Psychology, $87,228-245$.

Gilboa, E., \& Revelle, W. (1994), Personality and the structure of affective responses. In S. Van Goozen, N. E. Van De Poll, \& J. A. Sargent, (Eds.), Essays on current issues in the field of emotion theory (pp. 134-159). Hillsdale, NJ: Erlbaum.

Gross, J. J. (1998). Antecedent- and response-focused emotion regulation: Divergent consequences for experience, expression, and physiology. Journal of Personality and Social Psychology, 74, 224-237.

Gross, J. J. (Ed.). (2007). Handbook of emotion regulation. New York: Guilford Press.

Hemenover, S. H. (2003). Individual differences in rate of affect change: Studies in affective chronometry. Journal of Personality and Social Psychology, 85, 121-131.

Kahneman, D., Krueger, A. B., Schkade, D. A., Schwartz, N., \& Stone, A. A. (2004). A survey method for characterizing daily life experience: The day reconstruction method. Science, 306, 1776-1780.

Langston, C. A. (1994). Capitalizing on and coping with daily-life events: Expressive responses to positive events. Journal of Personality and Social Psychology, 67, 1112-1125.

McLaughlin, K. A., Borkovec, T. D., \& Sibrava, N. J. (2007). The effects of worry and rumination on affect states and cognitive activity. Behavior Therapy, 38, 23-28.

Morrow, J., \& Nolen-Hoeksema, S. (1990). Effects of responses to depression on the remediation of depressive affect. Journal of Personality and Social Psychology, 58, 519-527. 
Nezlek, J. B., \& Kuppens, P. (2008). Regulating positive and negative emotions in daily life. Journal of Personality, 76, 561-579.

Ray, R. D., Wilhelm, F. H., \& Gross, J. J. (2008). All in the mind's eye? Anger rumination and reappraisal. Journal of Personality and Social Psychology, 94, 133-145.

Rimé, B., Mesquita, B., Philippot, P., \& Boca, S. (1991). Beyond the emotional event: Six studies on the social sharing of emotion. Cognition and Emotion, 5, 435-465.

Rimé, B. (2009). Emotion elicits the social sharing of emotion: Theory and empirical review. Emotion Review, 1, 60-85.

Rude, S. S., Maestas, K. L., \& Neff, K. (2007). Paying attention to distress: What's wrong with rumination? Cognition and Emotion, 21, 843-864.

Russell, J. A., \& Carroll, J. M. (1999). On the bipolarity of positive and negative affect. Psychological Bulletin, 125, 3-30.

Russell, J. A., \& Feldmann-Barrett, L. (1999). Core affect, prototypical emotional episodes, and other things called emotion: Dissecting the elephant. Journal of Personality and Social Psychology, 76, 805-819.

Sbarra, D. A. (2006). Predicting the onset of emotional recovery following nonmarital relationship dissolution: Survival analyses of sadness and anger. Personality and Social Psychology Bulletin, 32, 298-312.

Scheff, T. J. (1979). Catharsis in healing, ritual, and drama. Berkeley, CA: University of California Press.

Scherer, K. R., Walbott, H. G., \& Summerfield, A. B. (1986). Experiencing emotions: A cross-cultural study. Cambridge, England: Cambridge University Press.

Schimmack, U. (2003). Affect measurement in experience sampling research. Journal of Happiness Studies, 4, 79-106.

Schimmack, U., Oishi, S., Diener, E., \& Suh, E. (2000). Facets of affective experiences: A framework for investigations of trait affect. Personality and Social Psychology Bulletin, 26, 655-668.

Singer, J. D., \& Willett, J. B. (2003). Applied longitudinal data analysis: Modeling change and event occurrence. New York: Oxford University Press.

Sonnemans, J. (1991). Structure and determinants of emotional intensity. Unpublished doctoral dissertation, University of Amsterdam.

Sonnemans, J., \& Frijda, N. (1994). The structure of subjective emotional intensity. Cognition and Emotion, 8, 329-350.

Sonnemans, J., \& Frijda, N. (1995). The determinants of subjective emotional intensity. Cognition and Emotion, 9, 483-506.

Verduyn, P., Delvaux, E., Van Coillie, H., Tuerlinckx, F., \& Van Mechelen, I. (2009a). Predicting the duration of emotional experience: Two experience sampling studies. Emotion, 9, 83-91.

Verduyn, P., Van Mechelen, I., Tuerlinckx, F., Meers, K., \& Van Coillie, H. (2009b). Intensity profiles of emotional experience over time. Cognition \& Emotion, 23, 1427-1443.

Wenzlaff, R. M., Wegner, D. M., \& Roper, D. W. (1988). Depression and mental control: The resurgence of unwanted negative thoughts. Journal of Personality and Social Psychology, 55, 882-892.

Zech, E., \& Rimé, B. (2005). Is talking about an emotional experience helpful? Effects on emotional recovery and perceived benefits. Clinical Psychology and Psychotherapy, 12, 270-287.

Received September 30, 2009

Revision received May 18, 2010

Accepted June 10, 2010

\section{E-Mail Notification of Your Latest Issue Online!}

Would you like to know when the next issue of your favorite APA journal will be available online? This service is now available to you. Sign up at http://notify.apa.org/ and you will be notified by e-mail when issues of interest to you become available! 